\title{
Graphene Nanoribbons Derived from the Unzipping of Carbon Nanotubes: Controlled Synthesis and Superior Lithium Storage Performance
}

\author{
Biwei Xiao, Xifei Li, Xia Li, Biqiong Wang, Craig Langford, Ruying Li, and Xueliang Sun* \\ Department of Mechanical and Materials Engineering, University of Western Ontario, London, ON, Canada N6A 5B9
}

Supporting Information

\begin{abstract}
Graphene nanoribbons (GNRs) from chemical unzipping of carbon nanotubes (CNTs) have been reported to be a suitable candidate for lithium ion battery materials, but very few of them focused on controlling GNRs with different unzipping levels. Here we present a study of GNRs with controlled unzipping level and the prevailing factors that affect the lithium storage performance at early and final unzipping level; besides, the effect of thermal reduction has been

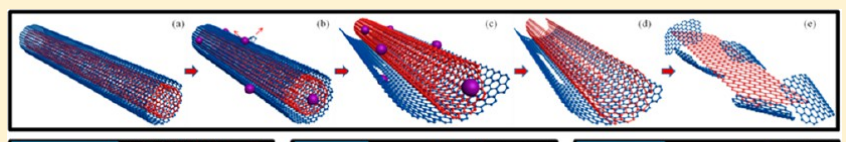
investigated. On the basis of Raman and BET surface area tests, we found that the unzipping of CNTs starts with surface etching and then proceeds to partial and full unzipping and finally fragmentation and aggregation. Galvanostatic charge-discharge reveals that defect increase is mainly responsible for the capacity enhancement at the early unzipping level; surface area drop is associated with the capacity fade at the final unzipping level. Surface functional groups can result in low electrical conductivity and therefore cause capacity drop within several cycles. The GNRs with controlled unzipping level display different electrochemical behaviors and thus can provide rational choices for researchers who are searching for desired functions using GNRs as additives in lithium ion batteries.
\end{abstract}

\section{INTRODUCTION}

Graphene is a two-dimensional monolayer of $\mathrm{sp}^{2}$-hybridized carbon atoms with a honeycomb lattice structure. It has attracted extensive investigations due to its unusual mechanical strength as well as excellent electrical and thermal stability. ${ }^{1}$ Carbon nanotubes (CNTs) are layers of graphene rolled up into seamless tubes; ${ }^{2}$ as a result, by unzipping carbon nanotubes, it is possible to obtain graphene nanoribbons (GNRs). ${ }^{3-6}$ Pioneered work was reported by Tour et al. ${ }^{7,8}$ By exposing the multiwalled CNTs in highly oxidizing solutions, they successfully achieved longitudinal cutting and unraveling of MWCNTs. Since then, many other strategies such as plasma etching, ${ }^{9,10}$ insertion and exfoliation, ${ }^{11}$ metal nanoparticle-catalyzed cutting, ${ }^{12,13}$ and mechanical sonication ${ }^{14}$ have been reported. The success of unzipping CNTs to GNRs has thereafter inspired the unzipping of other species of nanotubes such as boron nitride. ${ }^{15,16}$

In the past few decades, lithium ion batteries have become an emerging technology for high performance energy storage systems. ${ }^{17-19}$ Graphite is the currently commercialized anode material, but its inherent capacity is relatively low. ${ }^{20} \mathrm{CNTs}$ and graphene have been recognized as promising anode materials due to their unique high electrical conductivity and mechanical strength. ${ }^{21}$ The performance of CNTs and graphene strongly relies on their structural configuration such as surface area ${ }^{22,23}$ and defects. ${ }^{24-27}$ Therefore, much effort has been made to modify their pristine structures. Peralta et al. ${ }^{28}$ have calculated the lithium storage capability of graphene nanosheet (GNS), fullerenes, and GNRs based on density functional theory and revealed that the interaction between lithium and zigzag GNRs is
$50 \%$ stronger than that of GNS. This advantage contributes to the performance of lithium ion batteries as both anode materials and additives for electrode material composites. Besides the enhanced lithium storage property, the lithium diffusion coefficient has also been calculated in GNRs and was found to be elevated for up to 2 orders of magnitude compared with GNS. ${ }^{29}$ In addition, the robust mechanical property and enormous surface area of GNRs during lithiation have shown that GNRs can be an outstanding anode of MWCNTs in terms of durability and capacity. ${ }^{30}$ Despite this, the lithium storage capability of GNRs based on the unzipping level has not yet been studied, and the key factors that affect the performance are not clear.

In this study, we used modified Hummer's method ${ }^{31}$ to synthesize GNRs. By controlling the treatment time, we obtained GNRs with controlled unzipping level with changed surface areas, defect amounts, and functionalized surfaces. Moreover, we present a comprehensive study of the prevailing factor that influences the lithium storage capability of GNRs at different unzipping level. To the best of our knowledge, it is the first time systematically studying the morphology evolution of GNRs derived from CNTs and the lithium ion battery performance based on the GNRs series. It has been demonstrated that the number of defects increases right after exposure to oxidants; surface area changes gradually with the aggregation of GNRs

Received: November 2, 2013

Revised: December 19, 2013

Published: December 19, 2013 

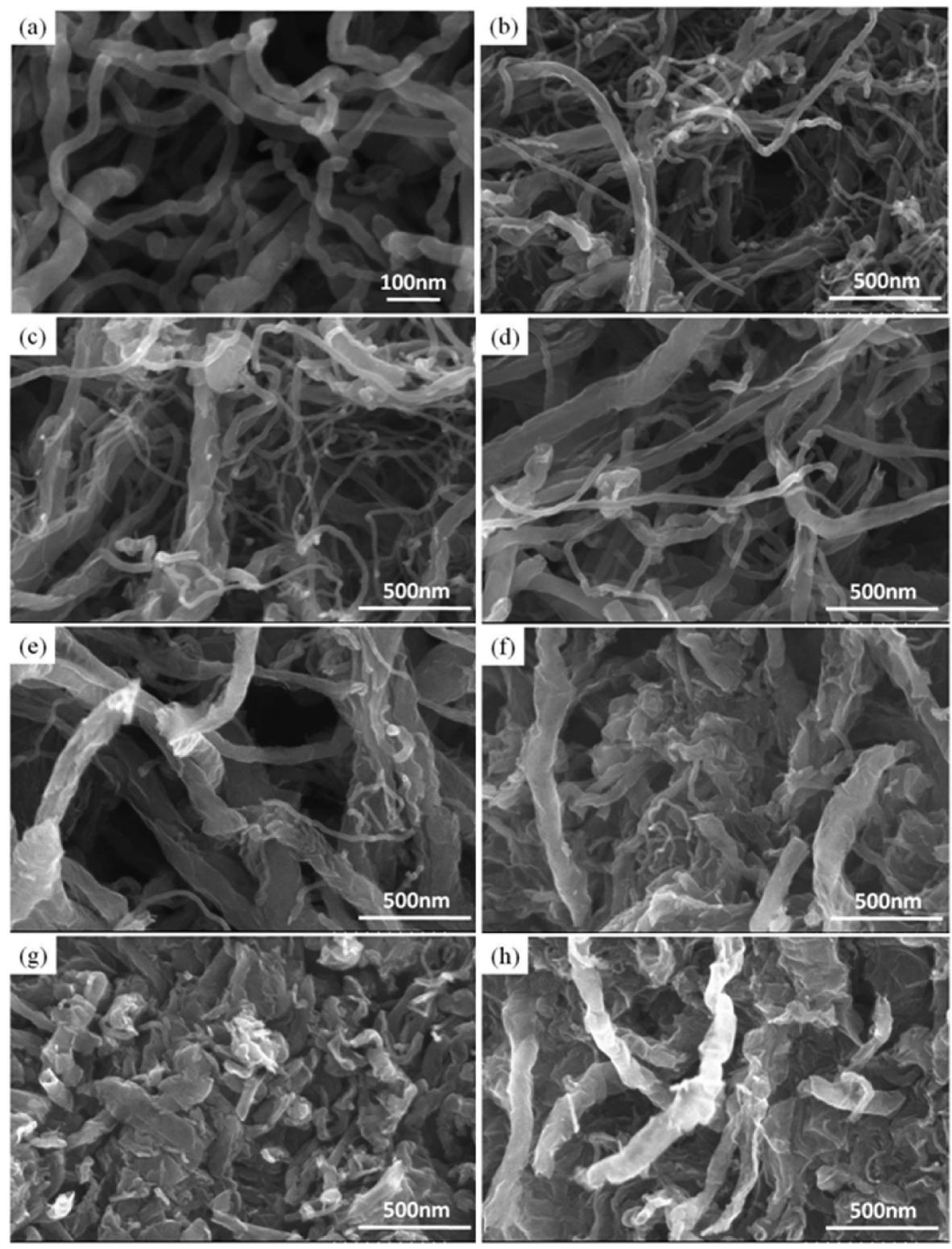

Figure 1. FE-SEM images of the pristine CNTs and GNRs series: (a) pristine CNTs; (b) GNRs-5min; (c) GNRs-30min; (d) GNRs-1h; (e) GNRs2.5h; (f) GNRs-5h; (g) GNRs-10h; (h) GNRs-20h.

under longer time due to the fragmentation of GNRs. The lithium storage capability of unzipped CNTs relies on different factors at different unzipping level. Defects are mainly responsible for the enhanced capacity at the early stage whereas surface area is associated with the capacity at high unzipping levels. Surface functional groups are found to enhance the discharge capacity; however, the increased impedance will lead to fading performance.

\section{EXPERIMENTAL SECTION}

2.1. Material Synthesis. In a typical unzipping process, 100 mg of MWCNTs (Shenzhen Nanotech., China) was dispersed in $3.4 \mathrm{~mL}$ of sulfuric acid $\left(\mathrm{H}_{2} \mathrm{SO}_{4}\right)$ (98\%, Aldrich) via strong ultrasonic agitation for $30 \mathrm{~min}$. The viscous solution was then placed in an ice bath under vigorous stirring, and $75 \mathrm{mg}$ of sodium nitrate $\left(\mathrm{NaNO}_{3}\right)$ (99.9\%, Aldrich) was subsequently added. After dissolving, $450 \mathrm{mg}$ of potassium permanganate $\left(\mathrm{KMnO}_{4}\right)(99.9 \%$, Aldrich) was slowly and carefully added into the viscous mixture. After a desired reaction time (specifically 5 min, $30 \mathrm{~min}, 1 \mathrm{~h}, 2.5 \mathrm{~h}, 5 \mathrm{~h}, 10 \mathrm{~h}$, and $20 \mathrm{~h}$ ), $20 \mathrm{~mL}$ of $5 \%$ sulfuric acid solution was poured into the liquid and left to cool. Next, 2 $\mathrm{mL}$ of hydrogen peroxide $\left(\mathrm{H}_{2} \mathrm{O}_{2}\right)(30 \%$, Aldrich $)$ was added into the solution in a dropwise manner until no more bubbles were released. After half an hour, the dark solution was centrifuged and thoroughly washed with $5 \%$ nitric acid three times and deionized water five times, then filtered, and dried in an oven at $90^{\circ} \mathrm{C}$ for 12 $\mathrm{h}$ under vacuum. The as-prepared GNRs contained high amounts of oxygen-containing functional groups (denoted as GONRs-oxidizing time) and are therefore annealed at $900{ }^{\circ} \mathrm{C}$ in Ar for reduction (denoted as GNRs-oxidizing time). To make the results more reliable, a reference was created with pristine CNTs which were treated in $30 \%$ nitric acid solution for $6 \mathrm{~h}$ to remove the catalysts.

2.2. Characterization Methods. The morphologies and structures of CNTs and GNRs were characterized by a Hitachi S4800 field emission scanning electronic microscopy (FESEM) equipped with an energy dispersive X-ray spectroscope (EDS), a Hitachi H-7000 transmission electron microscope (TEM), and a 

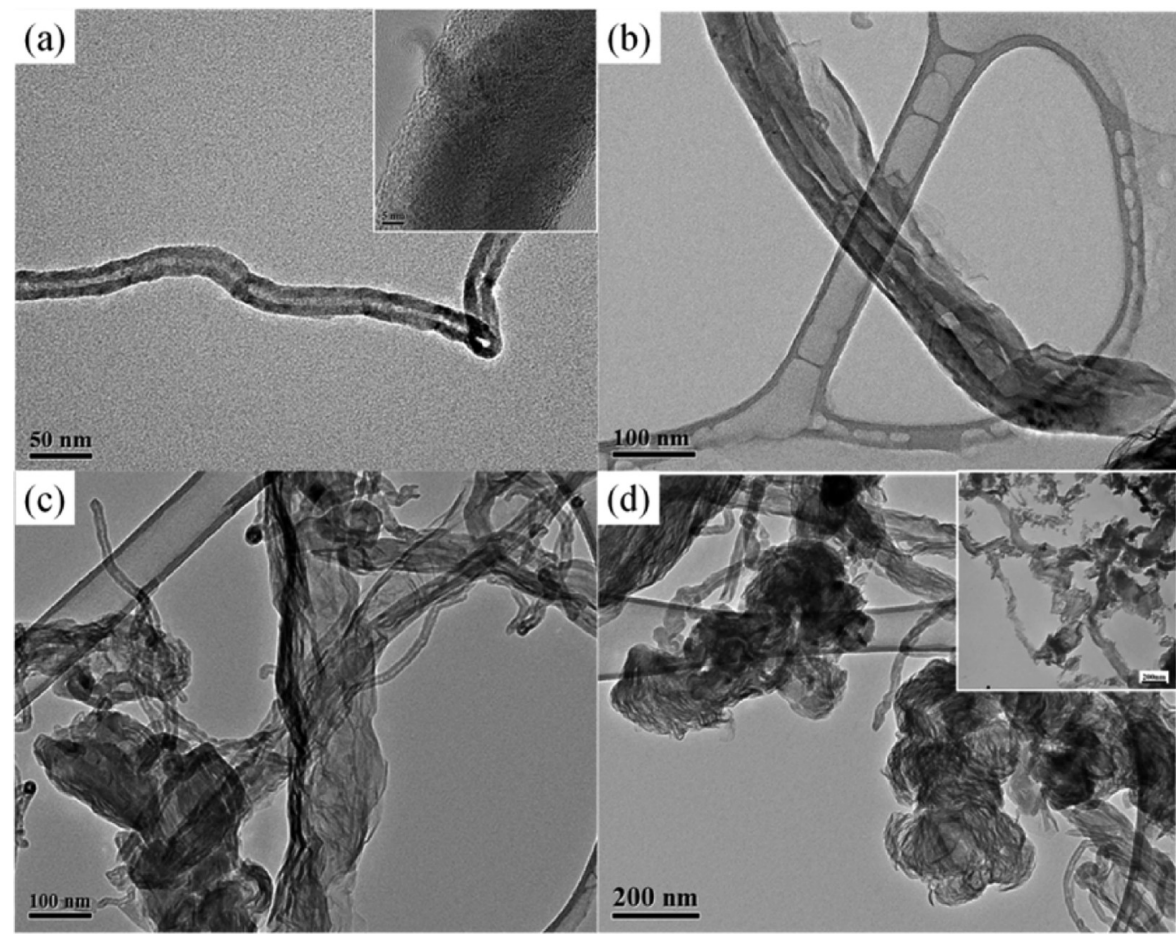

Figure 2. TEM images of CNTs during different unzipping stages: (a) etched CNTs; (b) partially unzipped CNTs; (c) fully unzipped CNTs; (d) stacked GNRs.

Scheme 1. Schematic Diagrams of (a) Pristine CNTs, (b) Etched CNTs, (c) Partially Unzipped CNTs, (d) Fully Unzipped CNTs, and (e) Stacked GNRs Fragments

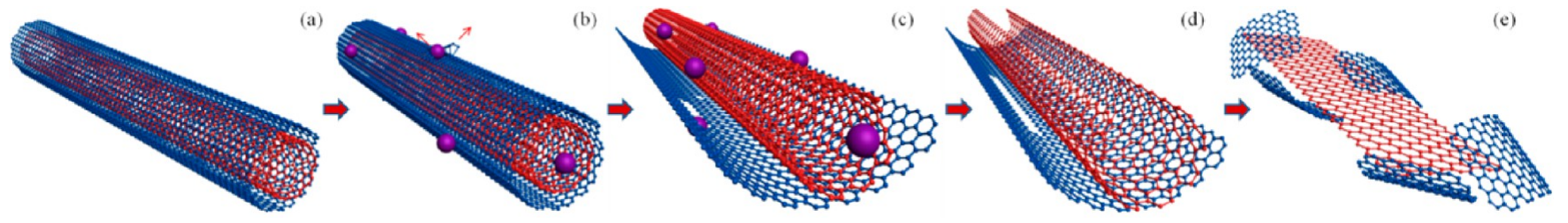

high-resolution transmission electron microscope (HRTEM, JEOL 2010F). Raman scattering (RS) spectra was obtained by a HORIBA Scientific LabRAM HR Raman spectrometer system with a $532.4 \mathrm{~nm}$ laser and optical microscope at room temperature; the intensity ratio of D and G band was calculated by integrating the areas after subtracting the baseline and applying Lorenzian fit. $\mathrm{N}_{2}$ adsorption/desorption isotherms were obtained by a Folio Micromeritics Tristar II surface area analyzer. Fourier transform infrared spectroscopy (FTIR) was obtained by a Nicolet 6700 FTIR spectrometer. Thermogravimetric analysis (TGA) was carried out on a TA SDT Q600 in an air atmosphere from room temperature to $700{ }^{\circ} \mathrm{C}$ at a rate of 10 ${ }^{\circ} \mathrm{C} / \mathrm{min}$.

2.3. Electrochemical Measurements. Pristine CNTs and GNRs were dispersed homogeneously in slurry with $10 \%$ poly(vinylidene fluoride) binder in $\mathrm{N}$-methylpyrrolidione (NMP) solvents. The slurry was subsequently casted onto a $\mathrm{Cu}$ foil as current collector and dried at $100{ }^{\circ} \mathrm{C}$ under vacuum overnight. The electrode was assembled in a glovebox with moisture and oxygen concentrations below 1 ppm. A CR-2325type coin cell with lithium metal as the counter electrode and Celgard 2400 as the separator was utilized. The electrolyte was composed of $1 \mathrm{M} \mathrm{LiPF}_{6}$ salt dissolved in ethylene carbonate:diethyl carbonate:ethylmethyl carbonate in a 1:1:1 volume ratio.
Both cyclic voltammetry $(\mathrm{CV})$ and electrochemical impedance spectra (EIS) were performed on a versatile multichannel potentiostat $3 / \mathrm{Z}$ (VMP3), with a scanning rate of $0.1 \mathrm{mV} / \mathrm{s}$ and a potential range of $0.1-3.0 \mathrm{~V}\left(\mathrm{vs}^{+} / \mathrm{Li}\right)$ at room temperature. Galvanostatical charge-discharge was performed on Arbin BT2000 at a current density of $100 \mathrm{~mA} / \mathrm{g}$ between 0.01 and $3.0 \mathrm{~V}\left(\mathrm{vs} \mathrm{Li}^{+} / \mathrm{Li}\right)$.

\section{RESULTS AND DISCUSSION}

Figure 1a shows the SEM image of pristine CNTs. Pristine CNTs have relatively uniform diameter distribution with an average value of $40 \mathrm{~nm}$. Figure $1 \mathrm{~b}-\mathrm{h}$ show time-dependent GNRs. It can be clearly observed that upon oxidizing the pristine CNTs present remarkable morphology evolution. CNTs have been successfully unzipped into U-shaped curved GNRs longitudinally. With increasing treatment time, the number of remaining CNTs become fewer and fewer. This is because CNTs with lower diameters are generally more stable in oxidizing conditions; ${ }^{7}$ therefore, the ratio of remaining CNTs is a direct indication of the level of oxidizing. After a $1 \mathrm{~h}$ treatment, about $50 \%$ of the CNTs were unzipped. In the case of a $5 \mathrm{~h}$ treatment, the CNTs have been fully unzipped. Continuous treatments lead to stacking of GNRs in the 10 and $20 \mathrm{~h}$ samples.

TEM and HRTEM were carried out in order to investigate the morphology evolution at higher magnifications as shown in 

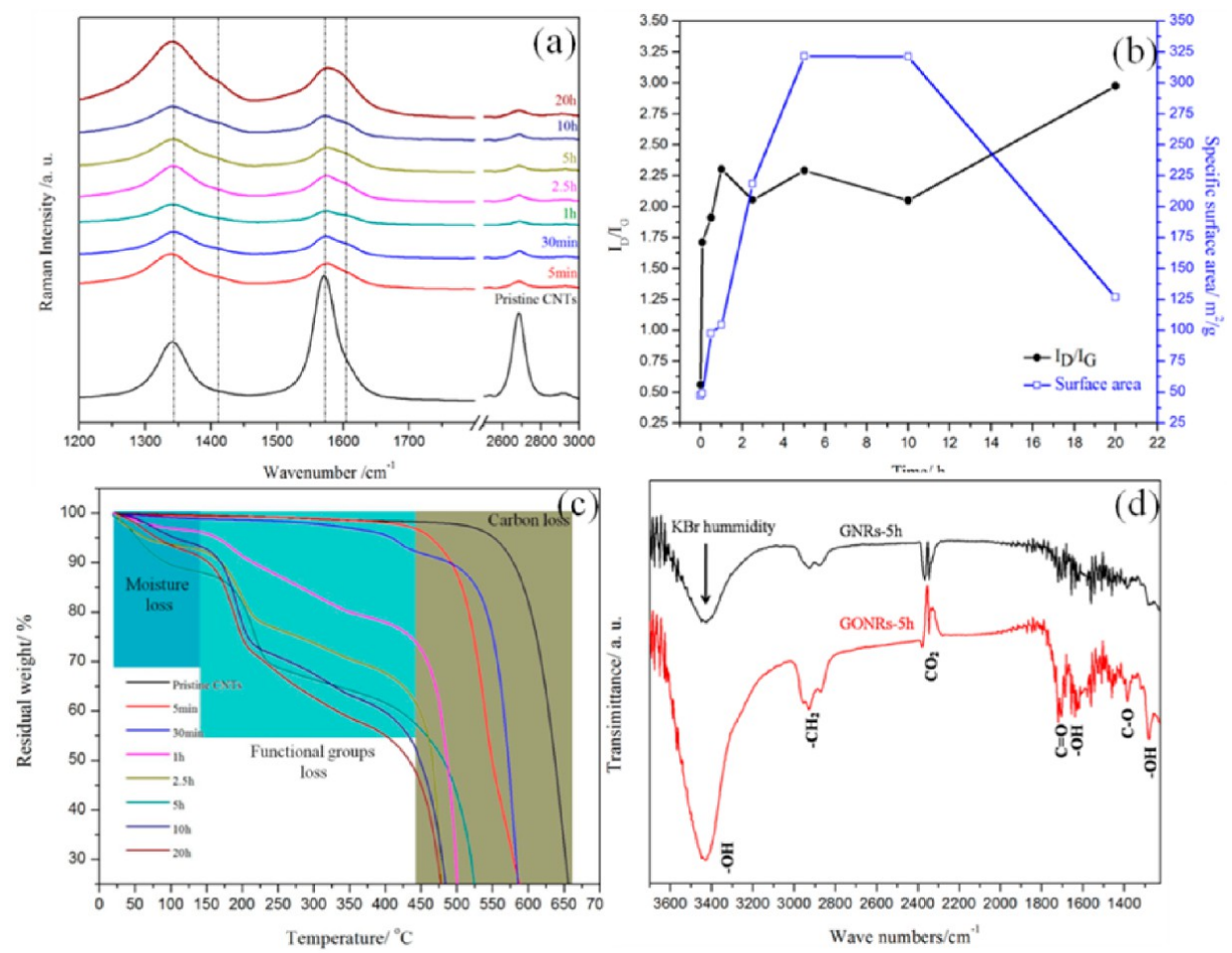

Figure 3. (a) Raman spectra of the pristine CNTs and GNRs. (b) Plot of the $I_{\mathrm{D}} / I_{\mathrm{G}}$ ratio and surface area. (c) TGA curves of pristine CNTs and GONRs. (d) FTIR of GNRs-oxide-5h and GNRs-5h.

Figure $2 \mathrm{a}-\mathrm{d}$. It can be seen that the unzipping of CNTs can be divided into four stages, namely surface etching, partially unzipping, fully unzipping, and aggregation. To better illustrate the process, we have proposed a schematic diagram of different unzipping levels. Scheme 1a shows pristine CNTs. Scheme $1 \mathrm{~b}$ is mostly observed in GNRs-5min, where the CNT has been etched with some cracks on the surface, however, the tubular structure still remains. HRTEM image Figure 2(b inset) clearly displays the etched layer of CNTs. It can be predicted that the cracks are generated on defect sites and the unzipping starts from these structures. Partially unzipped CNTs displayed in Scheme 1c reveal that the CNT is unwrapped layer by layer. With increasing time, the CNTs are fully unzipped longitudinally. However, extra treatment would produce fragmentation. As shown in Figure 2d, inset, and Scheme 1e, the GNRs have turned into flat structures, but the length decreases a lot in comparison with Figure $2 a-c$. At this stage, other fragments are also formed besides the ribbonlike structure. Severe aggregations can be observed in Figure 2d. The defects generated by initial unzipping are highly active sites for further attack of potassium permanganate, thus forming fragments. The fragments of GNRs are mainly responsible for the stacking found in Figure 1g,h.

Figure 3a shows the Raman spectra of pristine CNTs and GNRs. It can be observed that the pristine CNTs feature three obvious peaks at $1340.4,1572.1$, and $2683.1 \mathrm{~cm}^{-1}$. The peaks correspond to the $\mathrm{D}$ band induced by disordered defect, the $\mathrm{G}$ band caused by $\mathrm{sp}^{2}$ carbon vibration, and the $2 \mathrm{D}$ band of secondorder Raman scattering process, respectively. ${ }^{32}$ The intensity ratio of the $\mathrm{D}$ and $\mathrm{G}$ bands of pristine CNTs is 0.56 , indicating that the pristine CNTs are mostly $\mathrm{sp}^{2}$ hybridized. Besides the small amount of defect, amorphous carbon which is $\mathrm{sp}^{3}$ hybridized is also responsible for the $\mathrm{D}$ band intensity. The unzipping of CNTs creates new peaks both for D and G band at 1461.8 and $1610.4 \mathrm{~cm}^{-1}$, respectively; this can be ascribed to the vibration of graphite layers exfoliated from the CNTs. ${ }^{32}$ The $2 \mathrm{D}$ peak becomes much weaker as the unzipping proceeds as its peak intensity strongly relies on the $\mathrm{D}$ band intensity because of the defect-induced renormalization of electron and phonon energies. $^{33,34}$ Therefore, the increase of the $\mathrm{D}$ band intensity inevitably induces the drop of the $2 \mathrm{D}$ band intensity.

Figure $3 \mathrm{~b}$ displays a plot of the $I_{\mathrm{D}} / I_{\mathrm{G}}$ ratio calculated by the intensity of $\mathrm{D}$ and $\mathrm{G}$ bands. It can be seen that the defect amount increases significantly after the CNTs are exposed to oxidants. Further, longer treatment time does not cause severe change to the defect amount, and the values oscillate around 2.15 within the next $10 \mathrm{~h}$ of treatment. This implies that the defects are mostly created by the initial attack of the oxidants. Unzipping of CNTs does not exert strong impact on the formation of defects.

Figure $3 \mathrm{~b}$ also shows the plot of the Brunauer-EmmettTeller specific surface area of GNRs as a function of treated time. It can be seen that the surface area of pristine CNTs is $47.3 \mathrm{~m}^{2} / \mathrm{g}$. Within 5 min of treatment, the surface area is slightly increased to $49.1 \mathrm{~m}^{2} / \mathrm{g}$, which is in contrast with the sudden increase of defect. This reveals that the CNTs are likely to be etched on the surface at the early steps without damage of the tubular structures. The surface area is highest when the treatment time is $5 \mathrm{~h}$ with a value of $321.6 \mathrm{~m}^{2} / \mathrm{g}$; however, when the treatment time increased up to $10 \mathrm{~h}$, the surface area slightly decreased to $321.1 \mathrm{~m}^{2} / \mathrm{g}$. The balance of surface area at GNRs-5h and GNRs-10h implies that within this period of time unzipping and fragmentation-induced stacking happen simultaneously. When further reaction occurs at $20 \mathrm{~h}$, the surface area dramatically drops to $126.6 \mathrm{~m}^{2} / \mathrm{g}$, which indicates that unzipping has been mostly finished. But during this stage, the formation of GNRs fragments exceeds that of unzipping. The surface area change further shows that the fragmentation at 10 and $20 \mathrm{~h}$ is the reason for the surface area drop. 

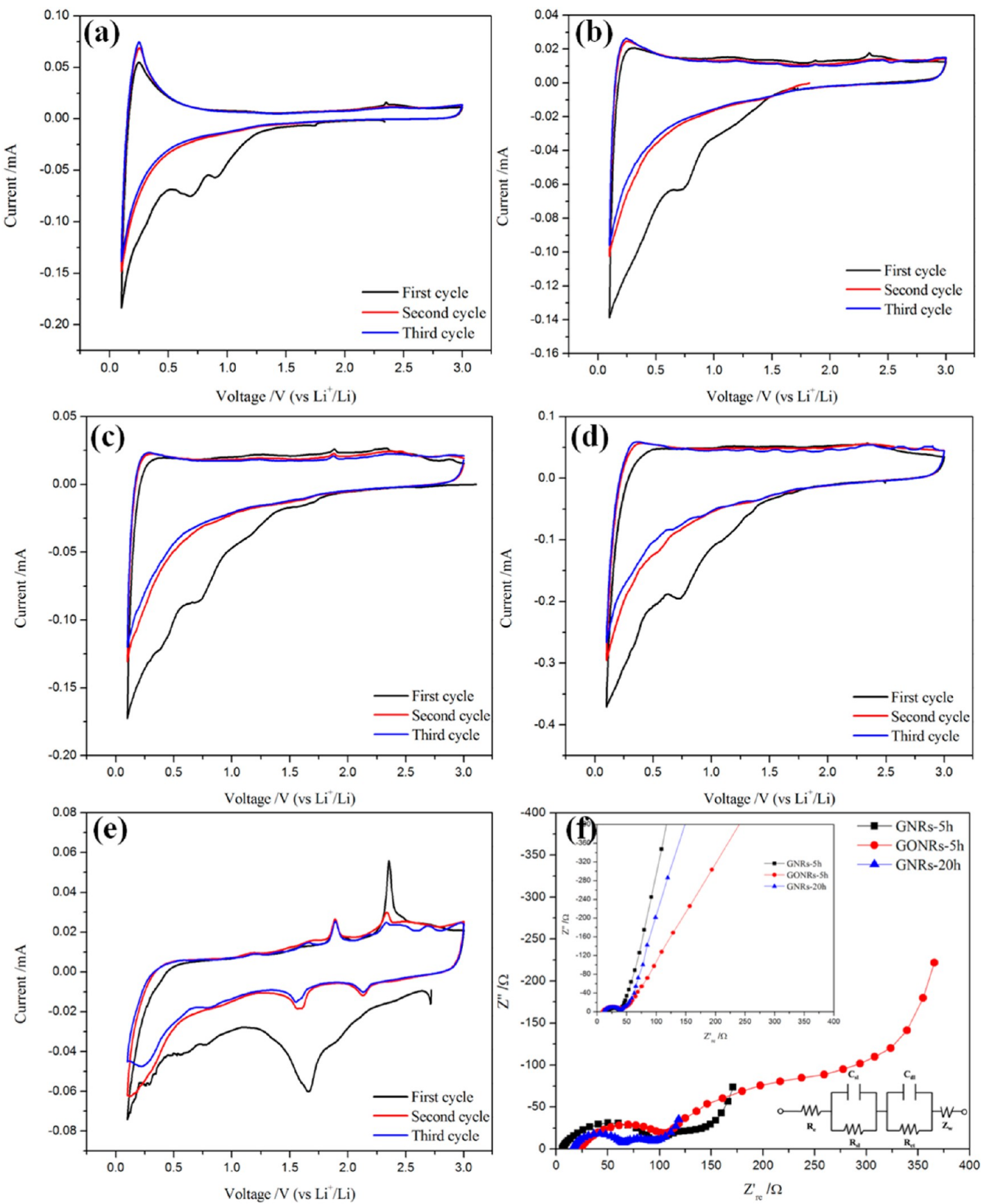

Figure 4. Cyclic voltammogram profiles of (a) pristine CNTs, (b) GNRs-5min, (c) GNRs-5h, (d) GNRs-20h, and (e) GONRs-5h as anodes at a scan rate of $0.1 \mathrm{mV} / \mathrm{s}$ in a voltage range of 0.1-3.00 V. (f) Electrochemical impedance spectra of GNRs-5h and GONRs-5h.

The thermal stability of the pristine CNTs and GONRs was confirmed by TGA shown in Figure $3 \mathrm{c}$. The initial weight loss below $100{ }^{\circ} \mathrm{C}$ is due to adsorbed moisture. Then, most of the GONRs display a weight loss from 150 to $450{ }^{\circ} \mathrm{C}$, which corresponds to the surface functional groups. The oxygencontaining groups are generally released as $\mathrm{CO}_{2}$. With higher oxidation level, the GONRs become less thermally stable, implying that the surface functional group magnitude increases 

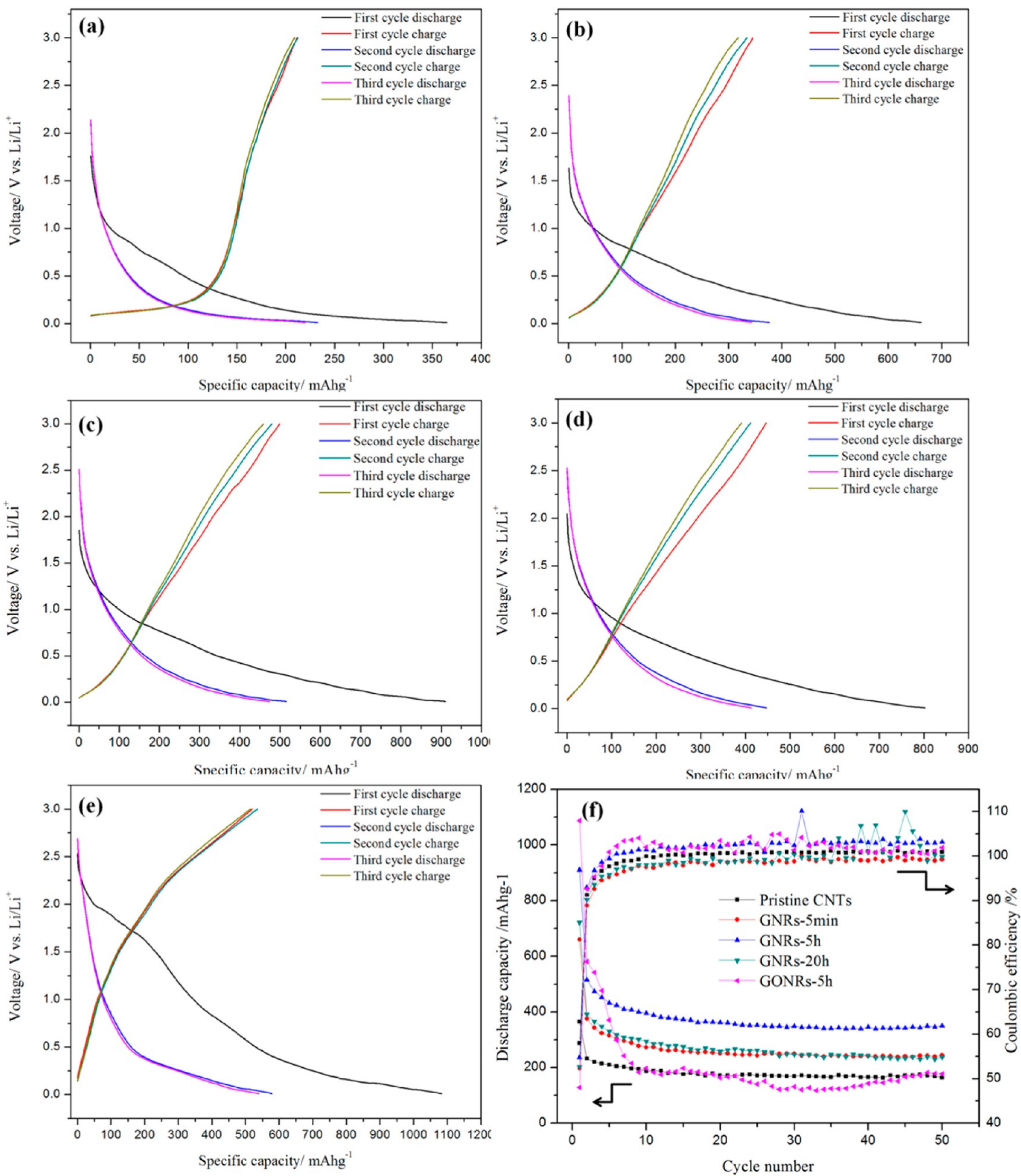

Figure 5. Charge/discharge profiles of (a) pristine CNTs, (b) GNRs-5min, (c) GNRs-5h, (d) GNRs-20h, and (e) GONRs-5h with a current density of $100 \mathrm{~mA} / \mathrm{g}$. (f) Discharge capacity and Coulombic efficiency versus cycle numbers of pristine CNTs and GNRs/GONRs at a current density of $100 \mathrm{~mA} /$ g.

with longer treatment. The final weight loss from 450 to $600{ }^{\circ} \mathrm{C}$ is because of the release of $\mathrm{CO}_{2}$ due to the burning of carbon.

The functional groups of GNRs-5h and GONRs-5h were confirmed via Fourier transform infrared spectroscopy (FTIR) as displayed in Figure 3d. The strong peaks at 3343 and $1630 \mathrm{~cm}^{-1}$ correspond to the hydroxyl stretching related to the $-\mathrm{OH}$ group and adsorbed water. It can be observed that before reduction GONRs-5h contains a large amount of adsorbed water due to the hydrophilic nature. ${ }^{35,36}$ After reduction, the vibration of hydroxyl has been decreased a lot. The broad peak at $3343 \mathrm{~cm}^{-1}$ can be ascribed to the $\mathrm{KBr}$ humidity. ${ }^{37}$ The doublet peaks at 2927 and $2872 \mathrm{~cm}^{-1}$ are due to the symmetric and antisymmetric 
stretching vibrations of $-\mathrm{CH}_{2} \cdot{ }^{38}$ The existence of $-\mathrm{CH}_{2}$ indicates that that the carbon basal planes are well maintained. Two intense peaks at 1714 and $1380 \mathrm{~cm}^{-1}$ are found at GONRs$5 \mathrm{~h}$, corresponding to the $\mathrm{C}=\mathrm{O}$ and $\mathrm{C}-\mathrm{O}$ stretching vibrations of $\mathrm{COOH}$ groups. ${ }^{39}$ Another strong band at $1380 \mathrm{~cm}^{-1}$ can be assigned to the $\mathrm{O}-\mathrm{H}$ deformations of the $\mathrm{C}-\mathrm{OH}$ groups. ${ }^{40}$ FTIR has clearly revealed that the majority of the functional groups in the GONRs-5h sample are oxygen-containing groups such as hydroxyl and carboxyl. Upon thermal reduction, the functional groups have been removed and released in the form of $\mathrm{CO}_{2}$.

As has been shown in Figure 3a,b, the defect of GNRs at $5 \mathrm{~min}$ is much higher than that of pristine CNTs while there is no surface area change. Therefore, it is good reference material to compare the performance of GNRs-5min with pristine CNTs to study the effect of defects. On the other hand, as both the defect and surface area keep increasing at GNRs-5min to GNRs-5h, it is difficult to ascribe the performance change to any of these two single factors. Therefore, the samples of the GNRs-5h and GNRs-20h provide us to study the surface area effect while there is a drop in surface area whereas an increase in defect amount. Moreover, the comparison of the GONRs-5h and GNRs-5h could help investigate the impact of surface functional groups.

The electrochemical behaviors of pristine CNTs, GNRs-5min, $-5 \mathrm{~h}$, and $-20 \mathrm{~h}$, and GONRs-5h were characterized by cyclic voltammetry $(\mathrm{CV})$ and galvanostatic charge and discharge process. Figures $4 \mathrm{a}, \mathrm{b}$ and $5 \mathrm{a}, \mathrm{b}$ present the $\mathrm{CV}$ and charge/ discharge curves of pristine CNTs and GNRs-5min at the first three cycles. It can be observed that both CV curves display two typical peaks at the first cathodic scan. The peak at about $0.75 \mathrm{~V}$ corresponds to the irreversible formation of SEI due to the decomposition of electrolyte, ${ }^{41}$ which is mainly responsible for the initial irreversible capacity. ${ }^{42}$ This peak disappears in the second and third cycle, indicating that the SEI is very stable. Another peak at $0.1 \mathrm{~V}$ reveals the insertion of $\mathrm{Li}^{+}$into the graphite layers. During anodic scan, the sharp peaks at $0.24 \mathrm{~V}$ for pristine CNTs and $0.30 \mathrm{~V}$ for GNRs-5min are related to the deintercalation if $\mathrm{Li}^{+}$from the graphene layers. ${ }^{43}$ A weak peak is observed in GNRs-5min at $1.85 \mathrm{~V}$, which is consistent with the previous results of ball-milled CNTs, chemically etched CNTs, and nanodrilled CNTs as anodes ${ }^{4-46}$ where small holes are created on the tube walls or the tube caps have been removed. This allows more $\mathrm{Li}^{+}$extraction from the interior space of nanotubes. This shows a strong evidence that surface defects on CNTs provides more sites for lithium storage. The peak at $2.34 \mathrm{~V}$ is due to the absorption of the $\mathrm{Li}^{+}$on remaining functional groups. ${ }^{45}$ In the case of GNRs-5min, this peak only exists at the first cycle, implying that there is no consequent capacity contribution. The reversible discharge capacity has been increased from 232.5 to $375.8 \mathrm{mAh} / \mathrm{g}$; this significant increase is due to the sudden increase of defect, which allows to accommodate more lithium. ${ }^{47}$

Figures $4 c, d$ and $5 c, d$ depict the $\mathrm{CV}$ and charge/discharge curves of GNRs-5h and GNRs-20h. Similar with that of GNRs$5 \mathrm{~min}$, a reversible peak at $1.85 \mathrm{~V}$ has been observed in GNRs-5h whereas there is no such peak in GNRs-20h. This change reveals that the tubular structure has been totally destroyed in GNRs20h, which is consistent with the SEM and TEM images that the GNRs have been turned into fragments. The oxidation peaks related to the extraction of $\mathrm{Li}^{+}$from graphite layers are located at 0.35 and $0.52 \mathrm{~V}$ for GNRs-5h and GNRs-20h, respectively. The charge/discharge curves show that GNRs-5h delivers much higher capacity than GNRs-20h. In the case of pristine CNTs and
GNRs-5min, defects are mainly responsible for the capacity increase; however, in the case of GNRs-5h and GNRs-20h, the discharge capacity decreases from 515.5 to $391.5 \mathrm{mAh} / \mathrm{g}$ even if there is an increase of defect amount. Realizing that the defect amount increase can possibly induce lower electrical conductivity for carbon materials, we conducted studies of electrochemical impedance spectroscopy (EIS) on GNRs-5h and GNRs-20h. As shown in Figure 4f, GNRs-5h and GNRs-20h at open circuit voltage (inset) display one semicircle and one straight line. The impedance of GNRs-5h is similar to GNRs-20h at open circuit voltage. In contrast to the EIS at open circuit voltage, the EIS at $0.01 \mathrm{~V}$ displays two semicircles at high and medium frequency region. To better illustrate the impedance behavior, we proposed a modified equivalent circuit in the inset of Figure $4 \mathrm{f} . R_{\mathrm{e}}$ stands for the electrolyte Ohmic resistance. The high-frequency semicircle can be ascribed to the resistance of SEI with the migration of $\mathrm{Li}^{+}$and interfacial capacitance $\left(C_{\mathrm{sl}}\right)$ related to $R_{\mathrm{sl}}$. The semicircle in medium-frequency region can be assigned to the charge-transfer resistance $\left(R_{\mathrm{ct}}\right)$ and the double-layer capacitance $\left(C_{\mathrm{dl}}\right) . W$ denotes the finite length Warburg impedance corresponding to the solid-state diffusion. Through the simulated equivalent circuit, we found that the charge transfer resistances of GNRs-5h and GNRs-20h are 21.9 and $22.2 \Omega$, respectively, indicating that the electrical conductivity of GNRs$20 \mathrm{~h}$ was not seriously affected by the presence of defects. Given the similar electrical conductivities of GNRs-5h and GNRs-20h, we therefore ascribe the capacity fade to the drop of surface area. High surface area allows for a high contact area between electrode and electrolyte to form SEI film by consuming a considerable amount of $\mathrm{Li}^{+}$. This leads to a high capacity at the first discharge. ${ }^{48}$ Despite the consumption of $\mathrm{Li}^{+}$, the high surface area provides much more free sites for the storage of $\mathrm{Li}^{+}{ }^{49}$ therefore, the reversible capacity of GNRs-5h is much higher than that of GNRs-20h.

Figures $4 \mathrm{e}$ and 5e display the $\mathrm{CV}$ and charge/discharge curves of GONRs-5h. By comparing with Figures $4 c$ and 5c (GNRs-5h), one can find two obvious redox peaks. The cathodic peaks located at the potential of $\mathrm{Li}^{+}$extraction from interior space ( 1.88 $\mathrm{V})$ and functional groups $(2.37 \mathrm{~V})$ are reversible during cycling. The redox peaks at 1.88 and $1.60 \mathrm{~V}$ correspond to the extraction of $\mathrm{Li}^{+}$from interior space of nanotubes. This peak is reversible and much more intense than GNRs-5h, but this observation remains unclear which possibly results from the self-repair effect during annealing. ${ }^{50}$ Another redox peaks at 2.37 and $2.13 \mathrm{~V}$ are assigned to the adsorption and extraction of $\mathrm{Li}^{+}$onto surface functional groups. It can be clearly seen that the oxidizing peak is very intense at the first cycle and becomes gradually weaker at the second and third cycles. It indicates that the adsorption of $\mathrm{Li}^{+}$on functional groups have a big contribution to the irreversible capacity. However, quite similar to the SEI film, the $\mathrm{Li}^{+}$are consumed rather than stored. The charge/discharge curves display two extra voltage plateaus, corresponding to the redox peaks of the functional groups. The curves also confirm the high irreversible discharge capacity of GONRs at $1088.0 \mathrm{mAh} / \mathrm{g}$ compared with GNR-5h (910.4 mAh/g).

Figure $5 f$ shows the cyclability of the performance and the Coulombic efficiencies of each sample. It is worthwhile to mention that the Coulombic efficiencies of GNRs-5h and GONRs-5h exceed $100 \%$. We believe this is caused by the double-layer capacitance enhanced by the side effect of high surface area. ${ }^{51,52}$ It can be seen that the GONRs-5h displays a higher reversible capacity in the first five cycles than other samples, but the stability is very poor, even lower than pristine 
Scheme 2. Schematic Diagrams of Insertion of Lithium Ions into (a) CNTs, (b) GNRs, and (c) GONRs

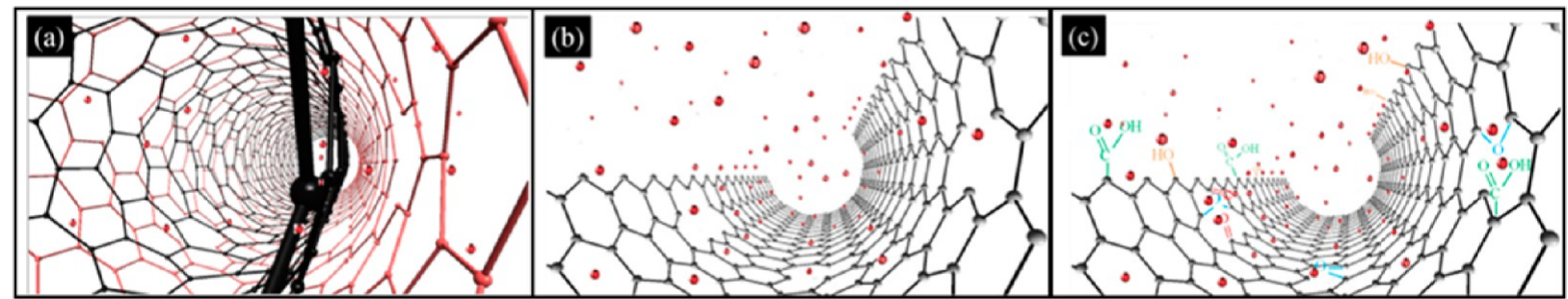

CNTs. Fahlman et al. ${ }^{53}$ also observed the similar performance of GONR. The high capacity of GONRs is ascribed to the stable Lirich SEI which prevents the electrolyte from further degradation. However, the poor cyclability of GONRs-5h may result from the decrease of electrical conductivity. To confirm this, electrochemical impedance spectroscopy (EIS) of GNRs-5h and GONRs-5h was conducted as shown in Figure $4 \mathrm{f}$, through the simulated equivalent circuit, we found that the charge transfer resistances of GNRs-5h and GONRs-5h are 21.9 and 82.0 $\Omega$, respectively, revealing that the electrical conductivity of GONRs$5 \mathrm{~h}$ is much lower than that of GNRs-5h. Therefore, we can conclude that the poor cyclability of GONRs-5h is because of the increased impedance due to the presence of surface functional groups.

To sum up, we have proposed the mechanisms of the enhanced capacity of GONRs and GNRs at each stage. Scheme $2 \mathrm{a}$ depicts the mechanism of $\mathrm{Li}^{+}$insertion into interlayers of MWCNTs at the early stage. Defects are created by oxidizing at the surface, thus allowing $\mathrm{Li}^{+}$migrate through the vacancies into the interlayers. This effect results in more sites for the accommodation of $\mathrm{Li}^{+}$, as the defect sites are generally highly active and therefore can absorb $\mathrm{Li}^{+}$. The mechanism of enhanced lithium storage capability at the later stage is shown in Scheme $2 b$. The noticeably increased surface area exposed to electrolyte provides more free sites to accommodate $\mathrm{Li}^{+}$while a thicker SEI film is formed. In the case of GONRs shown in Scheme 2c, the functional groups can absorb a remarkable amount of $\mathrm{Li}^{+}$, and along with the increased surface area, GONRs display very high irreversible capacity. ${ }^{54}$

\section{CONCLUSION}

In conclusion, the morphological evolution of GNRs derived from chemically unzipped CNTs has been divided into four steps according to the Raman spectra, TEM images, HRTEM images, and surface area. Specifically, the unzipping begins with chemical etching at the tube walls without severe damage to the tubular structure. Following etching results in partially and fully unzipped CNTs, however, continuous etching will lead to fragmentation of GNRs which is reflected as a drop of surface area due to aggregation.

We have studied the lithium storage performance of GNRs at different unzipping levels. The enhanced capacity at the early stage is mainly related to the obvious increase of defects. GNRs$5 \mathrm{~h}$, which has the highest surface area, delivers a reversible discharge capacity of over $500 \mathrm{mAh} / \mathrm{g}$. However, when the unzipping level comes to its final stage, the capacity drops noticeably due to the drop of surface area. Also, we have studied the functional groups effect on cycling performance of anodes, demonstrating that the functional groups can absorb a considerable amount of $\mathrm{Li}^{+}$which is mostly irreversible. The dropped cyclability of GONRs-5h is due to the decreased electrical conductivity. The systematic study on the lithium ion battery performance of GNRs with controlled unzipping levels can provide a strong reference for future utilization of GNRs and GNR composites.

\section{ASSOCIATED CONTENT}

\section{S Supporting Information}

Table showing the diameter distribution of pristine CNTs; elemental mapping of GNRs-5 $\mathrm{h}$ and GONRs- $5 \mathrm{~h}$. This material is available free of charge via the Internet at http://pubs.acs.org.

\section{AUTHOR INFORMATION}

\section{Corresponding Author}

*Tel +1 $5196612111 \times 87759 ; \mathrm{Fax}+1519661$ 3020; e-mail xsun@eng.uwo.ca (X.S.).

\section{Notes}

The authors declare no competing financial interest.

\section{ACKNOWLEDGMENTS}

This research was supported by the Natural Science and Engineering Research Council of Canada, the Canada Research Chair Program, the Canada Foundation for Innovation, and the University of Western Ontario.

\section{REFERENCES}

(1) Geim, A. K.; Novoselov, K. S. The Rise of Graphene. Nat. Mater. 2007, 6, 183-191.

(2) Iijima, S. Helical Microtubules of Graphiitic Carbon. Nature 1991, 354 (7), 56-58.

(3) Al-Aqtash, N.; Li, H.; Wang, L.; Mei, W.-N.; Sabirianov, R. F. Electromechanical Switching in Graphene Nanoribbons. Carbon 2013, $51,102-109$.

(4) Wang, Z.; Hu, H.; Zeng, H. The Electronic Properties of Graphene Nanoribbons with Boron/Nitrogen Codoping. Appl. Phys. Lett. 2010, 96 (24), 243110-243112.

(5) Bai, J.; Huang, Y. Fabrication and Electrical Properties of Graphene Nanoribbons. Mater. Sci. Eng., R 2010, 70 (3-6), 341-353.

(6) Chen, L.; Hernandez, Y.; Feng, X.; Müllen, K. From Nanographene and Graphene Nanoribbons to Graphene Sheets: Chemical Synthesis. Angew. Chem., Int. Ed. 2012, 51 (31), 7640-7654.

(7) Kosynkin, D. V.; Higginbotham, A. L.; Sinitskii, A.; Lomeda, J. R.; Dimiev, A.; Price, B. K.; Tour, J. M. Longitudinal Unzipping of Carbon Nanotubes to Form Graphene. Nature 2009, 458, 872-876.

(8) Higginbotham, A. L.; Kosynkin, D. V.; Sinitskii, A.; Sun, Z.; Tour, J. M. Lower-Defect Graphene Oxide Nanoribbons from Multiwalled Carbon Nanotubes. ACS Nano 2010, 4 (4), 2059-2069.

(9) Jiao, L.; Zhang, L.; Wang, X.; Diankov, G.; Dai, H. Narrow Graphene Nanoribbons from Carbon Nanotubes. Nature 2009, 458, $877-880$.

(10) Mohammadi, S.; Kolahdouz, Z.; Mohajerzadeh, S. Hydrogenation-Assisted Unzipping of Carbon Nanotubes to Realize Graphene Nano-Sheets. J. Mater. Chem. C 2013, 1 (7), 1309.

(11) Cano-Marquez, A. G.; Rodriguez-Macias, F. J.; Campos-Delgado, J.; Espinosa-Gonzalez, C. G.; Tristan-Lopez, F.; Ramirez-Gonzalez, D.; Cullen, D. A.; Smith, D. J.; Terrones, M.; Vega-Cantu, Y. I. Ex-MWNTs: 
Graphene Sheets and Ribbons Produced by Lithium Intercalation and Exfoliation of Carbon Nanotubes. Nano Lett. 2009, 9 (4), 1527-1533.

(12) Parashar, U. K.; Bhandari, S.; Srivastava, R. K.; Jariwala, D.; Srivastava, A. Single Step Synthesis of Graphene Nanoribbons by Catalyst Particle Size Dependent Cutting of Multiwalled Carbon Nanotubes. Nanoscale 2011, 3 (9), 3876-3882.

(13) Elías, A. L.; Botello-Méndez, A. R.; Meneses-Rodríguez, D.; González, V. J.; Ramirez-Gonzalez, D.; Ci, L.; Muñoz-Sandoval, E.; Ajayan, P. M.; Terrones, H.; Terrones, M. Longitudinal Cutting of Pure and Doped Carbon Nanotubes to Form Graphitic Nanoribbons Using Metal Clusters as Nanoscalpels. Nano Lett. 2010, 10 (2), 366-372.

(14) Jiao, L.; Wang, X.; Diankov, G.; Wang, H.; Dai, H. Facile Synthesis of High-Quality Graphene Nanoribbons. Nat. Nanotechnol. 2010, 5, 321-325.

(15) Erickson, K. J.; Gibb, A. L.; Sinitskii, A.; Rousseas, M.; Alem, N.; Tour, J. M.; Zettl, A. K. Longitudinal Splitting of Boron Nitride Nanotubes for the Facile Synthesis of High Quality Boron Nitride Nanoribbons. Nano Lett. 2011, 11 (8), 3221-3226.

(16) Zeng, H.; Zhi, C.; Zhang, Z.; Wei, X.; Wang, X.; Guo, W.; Bando, Y.; Golberg, D. "White Graphenes": Boron Nitride Nanoribbons Via Boron Nitride Nanotube Unwrapping. Nano Lett. 2010, 10 (12), 50495055

(17) Yoshino, A. The Birth of the Lithium-Ion Battery. Angew. Chem., Int. Ed. 2012, 51, 2-5.

(18) Tarascon, J. M. Key Challenges in Future Li-Battery Research. Philos. Trans. R. Soc., A 2010, 368 (1923), 3227-3241.

(19) Scrosati, B.; Hassoun, J.; Sun, Y.-K. Lithium-Ion Batteries. A Look into the Future. Energy Environ. Sci. 2011, 4 (9), 3287-3295.

(20) Simon, B.; Flandrois, S.; Guerin, K.; Fevrier-Bouvier, A.; Teulat, I.; Biensan, P. On the Choice of Graphite for Lithium Ion Batteries. J. Power Sources 1999, 81 (82), 312-316.

(21) de las Casas, C.; Li, W. A Review of Application of Carbon Nanotubes for Lithium Ion Battery Anode Material. J. Power Sources 2012, 208, 74-85.

(22) Liu, C.; Li, F.; Ma, L.-P.; Cheng, H.-M. Advanced Materials for Energy Storage. Adv. Mater. 2010, 22 (8), E28-E62.

(23) Valencia, F.; Romero, A. H.; Acilotto, F.; Silvestrelli, P. L. Lithium Adsorption on Graphite from Density Functional Theory Calculations. J. Phys. Chem. B 2006, 110, 14832-14841.

(24) Li, X.; Liu, J.; Zhang, Y.; Li, Y.; Liu, H.; Meng, X.; Yang, J.; Geng, D.; Wang, D.; Li, R.; Sun, X. High Concentration Nitrogen Doped Carbon Nanotube Anodes with Superior $\mathrm{Li}^{+}$Storage Performance for Lithium Rechargeable Battery Application. J. Power Sources 2012, 197, $238-245$.

(25) Zhou, L.-J; Hou, Z.-F.; Wu, L.-M. First-Principles Study of Lithium Adsorption and Diffusion on Graphene with Point Defects. J. Phys. Chem. C 2012, 116, 21780-21787.

(26) Yang, J.; Wang, J.; Li, X.; Wang, D.; Liu, J.; Liang, G.; Gauthier, M.; Li, Y.; Geng, D.; Li, R.; Sun, X. Hierarchically Porous $\mathrm{LiFePO}_{4} /$ Nitrogen-Doped Carbon Nanotubes Composite as a Cathode for Lithium Ion Batteries. J. Mater. Chem. 2012, 22, 7537-7543.

(27) Wu, Z.-S.; Ren, W.; Xu, L.; Li, F.; Cheng, H.-M. Doped Graphene Sheets as Anode Materials with Super High Rate and Large Capacity for Lithium Ion Batteries. ACS Nano 2011, 5 (7), 5463-5471.

(28) Uthaisar, C.; Barone, V.; Peralta, J. E. Lithium Adsorption on Zigzag Graphene Nanoribbons. J. Appl. Phys. 2009, 106 (11), 113715113720.

(29) Uthaisar, C.; Barone, V. Edge Effects on the Characteristics of Li Diffusion in Graphene. Nano Lett. 2010, 10 (8), 2838-2842.

(30) Liu, X. H.; Wang, J. W.; Liu, Y.; Zheng, H.; Kushima, A.; Huang, S.; Zhu, T.; Mao, S. X.; Li, J.; Zhang, S.; Lu, W.; Tour, J. M.; Huang, J. Y. In Situ Transmission Electron Microscopy of Electrochemical Lithiation, Delithiation and Deformation of Individual Graphene Nanoribbons. Carbon 2012, 50 (10), 3836-3844.

(31) Hummers, W. S.; Offeman, R. E. Preparation of Graphitic Oxide. J. Am. Chem. Soc. 1958, 80 (6), 1339-1339.

(32) Dresselhaus, M. S.; Dresselhaus, G.; Saito, R.; Jorio, A. Raman Spectroscopy of Carbon Nanotubes. Phys. Rep. 2005, 409 (2), 47-99.
(33) Maciel, I. O.; Anderson, N.; Pimenta, M. A.; Hartshuh, A.; Qian, H.; Terrones, M.; Terrones, H.; Campos-Delgado, J.; Rao, A. M.; Novotny, L.; Jorio, A. Electron and Phonon Renormalization near Charged Defects in Carbon Nanotubes. Nat. Mater. 2008, 7, 878-883.

(34) Dresselhaus, M. S.; Jorio, A.; Hofmann, M.; Dresselhaus, G.; Saito, R. Perspectives on Carbon Nanotubes and Graphene Raman Spectroscopy. Nano Lett. 2010, 10 (3), 751-758.

(35) Dimiev, A.; Kosynkin, D. V.; Alemany, L. B.; Chaguine, P.; Tour, J. M. Pristine Graphite Oxide. J. Am. Chem. Soc. 2012, 134 (5), 28152822.

(36) Pei, S.; Cheng, H.-M. The Reduction of Graphene Oxide. Carbon 2012, 50 (9), 3210-3228.

(37) Kaniyoor, A.; Baby, T. T.; Ramaprabhu, S. Graphene Synthesis Via Hydrogen Induced Low Temperature Exfoliation of Graphite Oxide. J. Mater. Chem. 2010, 20 (39), 8467-8469.

(38) Sun, X.; Liu, Z.; Welsher, K.; Robinson, J. T.; Goodwin, A.; Zaric, S.; Dai, H. Nano-Graphene Oxide for Cellular Imaging and Drug Delivery. Nano Res. 2008, 1, 203-212.

(39) Jeong, H.-K.; Colakerol, L.; Jin, M. H.; Glans, P.-A.; Smith, K. E.; Lee, Y. H. Unoccupied Electronic States in Graphite Oxides. Chem. Phys. Lett. 2008, 460, 499-502.

(40) Bourlinos, A. B.; Gournis, D.; Petridis, D.; Szabo, T.; Szeri, A.; Dekany, I. Graphite Oxide: Chemical Reduction to Graphite and Surface Modification with Primary Aliphatic Amines and Amino Acids. Langmuir 2003, 19, 6050-6055.

(41) Landi, B. J.; Ganter, M. J.; Schauerman, C. M.; Cress, C. D.; Raffaelle, R. P. Lithium Ion Capacity of Single Wall Carbon Nanotube Paper Electrodes. J. Phys. Chem. C 2008, 112 (19), 7509-7515.

(42) Chusid, O. Y.; Ely, Y. E.; Babai, M.; Carmeli, Y.; Aurbach, D. Electrochemical and Spectroscopic Studies of Carbon Electrodes in Lithium Battery Electrolyte Systems. J. Power Sources 1993, 43 (44), 4764.

(43) Landi, B. J.; Ganter, M. J.; Cress, C. D.; DiLeo, R. A.; Raffaelle, R. P. Carbon Nanotubes for Lithium Ion Batteries. Energy Environ. Sci. 2009, 2 (6), 638-654.

(44) Shimoda, H.; Gao, B.; Tang, X.; Kleinhammes, A.; Fleming, L.; $\mathrm{Wu}, \mathrm{Y}$;; Zhou, O. Lithium Intercalation into Opened Single-Wall Carbon Nanotubes: Storage Capacity and Electronic Properties. Phys. Rev. Lett. 2001, 88 (1), 015502-015505.

(45) Oktaviano, H. S.; Yamada, K.; Waki, K. Nano-Drilled Multiwalled Carbon Nanotubes: Characterizations and Application for LIB Anode Materials. J. Mater. Chem. 2012, 22 (48), 25167-25173.

(46) Eom, J. Y.; Kwon, H. Improved Lithium Insertion/Extraction Properties of Single-Walled Carbon Nanotubes by High-Energy Ball Milling. J. Mater. Res. 2008, 23 (9), 2458-2466.

(47) Yao, F.; Güneş, F.; Ta, H. Q.; Lee, S. M.; Chae, S. J.; Sheem, K. Y.; Cojocaru, C. S.; Xie, S. S.; Lee, Y. H. Diffusion Mechanism of Lithium Ion through Basal Plane of Layered Graphene. J. Am. Chem. Soc. 2012, $134(20), 8646-8654$.

(48) Béguin, F.; Chevallier, F.; Vix-Guterl, C.; Saadallah, S.; Bertagna, V.; Rouzaud, J. N.; Frackowiak, E. Correlation of the Irreversible Lithium Capacity with the Active Surface Area of Modified Carbons. Carbon 2005, 43 (10), 2160-2167.

(49) Xiang, H. F.; Li, Z. D.; Xie, K.; Jiang, J. Z.; Chen, J. J.; Lian, P. C.; Wu, J. S.; Yu, Y.; Wang, H. H. Graphene Sheets as Anode Materials for Li-Ion Batteries: Preparation, Structure, Electrochemical Properties and Mechanism for Lithium Storage. RSC Adv. 2012, 2 (17), 6792-6799.

(50) López, V.; Sundaram, R. S.; Gómez-Navarro, C.; Olea, D.; Burghard, M.; Gomez-Herrero, J.; Zamora, F.; Kern, K. Chemical Vapor Deposition Repair of Graphene Oxide: A Route to Highly-Conductive Graphene Monolayers. Adv. Mater. 2009, 21 (46), 4683-4686.

(51) Zhao, C.; Zheng, W.; Wang, X.; Zhang, H.; Cui, X.; Wang, H. Ultrahigh Capacitive Performance from Both $\mathrm{Co}(\mathrm{OH})_{2} /$ Graphene Electrode and $\mathrm{K}_{3} \mathrm{Fe}(\mathrm{CN})_{6}$ Electrolyte. Sci. Rep. 2013, 3, 2986.

(52) Wang, C.; Appleby, A. J.; Little, F. E. Electrochemical Impedance Study of Initial Lithium Ion Intercalation into Graphite Powders. Electrochim. Acta 2001, 46, 1793-1813. 
(53) Bhardwaj, T.; Antic, A.; Pavan, B.; Barone, V.; Fahlman, B. D. Enhanced Electrochemical Lithium Storage by Graphene Nanoribbons. J. Am. Chem. Soc. 2010, 132, 12556-12558.

(54) Lee, S. W.; Yabuuchi, N.; Gallant, B. M.; Chen, S.; Kim, B.-S.; Hammond, P. T.; Shao-Horn, Y. High-Power Lithium Batteries from Functionalized Carbon-Nanotube Electrodes. Nat. Nanotechnol. 2010, $5,531-537$. 